CARNETS OE Carnets de géographes

GÉOGRAPHES.

4 | 2012

Géographies critiques

\title{
Inégalités et pouvoirs au centre-ville du cap
}

Une nécessaire lecture critique

\section{Thomas Radovcic}

\section{(2) OpenEdition}

Journals

Édition électronique

URL : http://journals.openedition.org/cdg/1004

DOI : $10.4000 /$ cdg. 1004

ISSN : 2107-7266

Éditeur

UMR 245 - CESSMA

\section{Référence électronique}

Thomas Radovcic, «Inégalités et pouvoirs au centre-ville du cap », Carnets de géographes [En ligne],

4 | 2012, mis en ligne le 01 septembre 2012, consulté le 30 avril 2019. URL : http://

journals.openedition.org/cdg/1004; DOI : 10.4000/cdg.1004

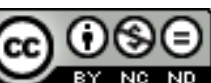

La revue Carnets de géographes est mise à disposition selon les termes de la Licence Creative Commons Attribution - Pas d'Utilisation Commerciale - Pas de Modification 4.0 International. 


\section{INEGALITES ET POUVOIRS AU CENTRE-VILLE DU CAP}

une nécessaire lecture critique

THOMAS RADOVCIC

Géographe indépendant

thomas.radovcic@gmail.com

\section{Note de l'auteur : une nécessaire lecture critique}

Cet article découle d'un travail de recherche effectué en 2010 et 2011 au cours de mes deux années de Master de géographie à l'Université Paris 1 Panthéon-Sorbonne. Les recherches de terrain dont rend compte cet article ont été réalisées grâce au soutien de la Région île-deFrance et de I'Institut Français d'Afrique du Sud. Toutefois, cet article n'engage que moi et non mon institution de formation ou celles qui m'ont financé.

Affirmer que la ville sud-africaine de l'apartheid était injuste est un pléonasme : une série de lois instaurées depuis $1948^{1}$ définissait jusqu'en 1991 la répartition spatiale de la population sur le territoire en fonction de groupes raciaux ${ }^{2}$ (grand apartheid) ainsi que les relations sociales entre individus de races différentes (apartheid mesquin) (Christopher, 1994; Fauvelle-Aymar, 2010).

Cependant, vingt ans après la chute du régime raciste et ségrégationniste, force est de constater que la société sud-africaine et les villes, y compris celle du Cap, sont toujours polarisées spatialement, socialement et racialement (Turok, 2001 ; Lemanski, 2007).

En dépit des dispositions de la Freedom Charter ${ }^{3}$ de 1955 et intégrées dans l'une des constitutions les plus progressistes du monde, les Africains ${ }^{4}$ et les Coloured discriminés

\footnotetext{
${ }^{1}$ L'adoption des lois racistes et ségrégationnistes instaurant l'apartheid ne constituent pas une rupture dans I'histoire sud-africaine mais une continuité avec l'époque coloniale et les premières décennies de l'indépendance sud-africaine (Gervais-Lambony, 2003).

${ }^{2}$ Alors que dans le contexte français, la « race " ne saurait être considérée comme pertinente tant son emploi est lié à l'idéologie raciste, les autorités et les chercheurs sud-africains utilisent ce terme sans pour autant adhérer à un quelconque déterminisme racial.

${ }^{3}$ La Freedom Charter est une déclaration de la South African Congress Alliance regroupant les organisations d'opposition au régime de l'apartheid. Cette charte, signée en 1955, regroupe les grands principes du droit qui seront en grande partie intégrés à la Constitution sud-africaine de 1996.

${ }^{4}$ Selon le Population Registration Act de 1950, chaque individu était versé dans un groupe racial : blanc, noir ou coloured, auquel sera rajouté plus tard le groupe des Indiens. Cette classification était fondée sur des critères d'apparence physique, d'appartenance à une communauté et de construction sociale. D'autre part, cette
} 
durant l'apartheid restent, encore aujourd'hui, les groupes de populations les plus désavantagés. A l'échelle sud-africaine, en 2006, plus de $90 \%$ des ménages blancs et indiens/asiatiques disposent d'eau courante à leur domicile contre $76 \%$ des ménages coloured et seulement $25 \%$ des ménages africains. Selon les critères de l'office national sudafricain de statistiques (Statistics South Africa), 81,5\% des ménages blancs disposent d'équipements domestiques de qualité (vivre dans un logement formel, où l'électricité est la source première de lumière, et l'électricité ou le gaz sont les sources principales d'énergie utilisées pour la cuisine, disposer de l'eau courante et de cabinet de toilette au domicile) contre $69,1 \%$ des ménages indiens/asiatiques, $49,2 \%$ des ménages coloured et $13,1 \%$ des ménages africains (Statistics South Africa, 2009).

Ces inégalités qui recoupent les catégories socio-raciales de l'apartheid sont renforcées depuis le milieu des années 1990 par l'ouverture internationale et la libéralisation économique ${ }^{5}$. Les chercheurs en sciences sociales travaillant sur l'Afrique du Sud en général et sur Le Cap en particulier ont utilisé et utilisent encore les catégories socio-raciales comme grille d'analyse. En effet, l'identité et la citadinité des Sud-Africains se sont construites dans le cadre de la politique de ségrégation imposée par l'apartheid, en réaction, voire en opposition à cette politique et dans les transformations récentes de la ville post-apartheid (Guillaume et Houssay-Holzschuch, 1998; Leildé, 2008). Ces catégories ne sont pas uniquement fondées sur des caractéristiques phénotypiques mais désignent des réalités culturelles (Western, 1981 ; Houssay-Holzschuch, 1998). D'autre part, même si la tendance actuelle est à une dépolarisation relative, les groupes de population recoupent en grande partie les classes sociales structurant la société sud-africaine. Par exemple, selon Statistics South Africa, à l'échelle des subcouncils ${ }^{6}$ en $2001^{7}$, on observe une corrélation positive significative entre un fort taux de chômage et une forte proportion d'Africains (89\%), et de même, entre une forte proportion de Blancs et revenu moyen des ménages élevé $(95 \%)^{8}$. La catégorisation raciale est, en outre, utilisée pour mettre en œuvre certaines politiques publiques afin de réduire et corriger les inégalités creusées durant l'apartheid, en particulier en termes d'accès à l'emploi (politique de Black Economic Empowerment, i.e. d'autonomisation des Noirs traduite par des mesures de discrimination positive notamment). Une analyse par le prisme des catégories raciales reste encore aujourd'hui

catégorisation était hiérarchisée : les Blancs était considérés comme le groupe dominant et jouissaient de nombreux privilèges alors que les Noirs étaient les plus défavorisés. Les Coloured avaient une situation plus ou moins intermédiaire, inférieure à celle des Indiens et supérieure à celle des Noirs. La qualité respective des espaces attribués à chaque groupe, même entre les townships noirs, coloured et indiens reflétait la hiérarchie des races.

Aujourd'hui, quatre groupes raciaux sont encore identifiés notamment dans la statistique officielle: Africains, Blancs, Coloured et Indiens/Asiatiques.

Le terme "noir » désigne les groupes de population discriminés durant l'apartheid: Africains, Coloured et Indiens/Asiatiques. Dans ce travail, j'utilise la terminologie sud-africaine officielle pour désigner les groupes de population.

${ }^{5}$ Les gouvernements sud-africains successifs ont effectué des choix stratégiques afin d'insérer le pays dans le jeu de la mondialisation et de stimuler la compétitivité économique du pays : promotion des exportations, libéralisation du commerce, dérégulation financière, rigueur fiscale sur les dépenses de santé et de logement, politique monétaire restrictive, baisse des taux d'imposition sur les entreprises, privatisation d'entreprises publiques, flexibilité sur le marché du travail. Cette libéralisation économique a renforcé les inégalités déjà criantes dans la société sud-africaine (Bond, 2000 ; McDonald, 2008)

${ }^{6}$ Les subcouncils sont l'une des subdivisions politiques de la Municipalité du Cap.

${ }^{7}$ Les données du recensement de 2011 ne sont pas encore publiées au moment de la rédaction de cet article.

${ }^{8}$ Statistics South Africa, recensement de la population, 2001. 
pertinente tant elle permet d'aborder des problématiques culturelles, sociales, politiques et d'aménagement du territoire aussi bien à l'échelle nationale que locale. D'un point de vue méthodologique, une telle approche est facilitée par la disponibilité d'un appareil statistique abondant et relativement fiable.

Mon expérience de citadin capetonien ${ }^{9}$ et de chercheur m'a cependant conduit à repenser cette démarche en considérant plus largement les rapports de domination et les relations de pouvoir en jeu dans la société urbaine au Cap.

J'ai choisi de concentrer mon travail de recherche sur le centre-ville du Cap, espace qui me semble emblématique des défis que connaît la métropole capetonienne post-apartheid. En effet, le centre-ville est à la fois un espace de concentration des richesses et des investissements (City of Cape Town, 2010) mais aussi un lieu ouvert partagé par l'ensemble des citadins, notamment en raison des opportunités d'emplois, des équipements de transports et de loisirs, ou encore de la concentration de commerces (Houssay-Holzschuch, 2010). Il est par ailleurs indéniable que ma connaissance intime du centre-ville a grandement guidé mon choix de ce terrain d'étude.

Les relations de pouvoir au centre-ville de la métropole dépassent largement le cadre des rapports de domination économique et politique entre classes socio-raciales. Afin de mener ma recherche de Master 1 sur le Greenmarket Square, une place du centre-ville fréquentée par les touristes internationaux et les citadins capetoniens, j'ai rencontré les acteurs en charge de sa gestion. Alors que mon projet initial qui visait à analyser les pratiques et les représentations des usagers de cet espace en fonction des catégories socio-raciales héritées de l'apartheid m'a permis d'identifier l'émergence d'une société urbaine cosmopolite et multiraciale, j'ai complété mon travail en prenant en compte la gouvernance des espaces publics du centre-ville. Le Greenmarket square a été, et est toujours, l'objet d'une lutte de pouvoir entre différents acteurs publics, parapublics et privés. L'évolution de ces rapports de pouvoir a pu façonner, au cours des trois dernières décennies, les usages de cette place, son urbanisme, les activités qui s'y sont développées et les profils des individus qui la pratiquent (Radovcic, 2010). Une mobilisation, parfois performative, de catégories raciales ne permet pas d'appréhender les relations de pouvoir plus diffuses ayant toutefois un impact sociospatial fondamental : une grille fondée sur les catégories raciales appelle nécessairement à une analyse en ces termes ne permettant pas d'appréhender d'autres mécanismes de domination. C'est ce constat qui m'a incité à poursuivre mes recherches sur la gouvernance urbaine du Cap à travers l'étude d'un projet de développement urbain mis en œuvre en centre-ville : The Fringe - Cape Town's Innovation District.

L'étude de ce programme m'a permis de mettre en évidence les contradictions de la ville post-apartheid entre recherche de compétitivité internationale fondée sur des investissements localisés et agenda développemental.

Comme la plupart des métropoles du monde, Le Cap cherche à se positionner dans la compétition internationale entre les villes. Le contexte d'internationalisation des échanges

\footnotetext{
9 J'ai pour la première fois vécu au Cap en 2005 à l'occasion d'un accord d'échange universitaire avant de m'y installer et d'y travailler pendant un an en 2007-2008. Le centre-ville du Cap a donc d'abord été le cadre de ma vie quotidienne (lieu de résidence, de travail et de loisirs) avant d'être un terrain de recherche.
} 
privilégie certaines villes comme lieux de croissance économique. Afin d'attirer des investissements et des travailleurs de plus en plus mobiles, les villes entrent en concurrence les unes avec les autres en faisant valoir les avantages comparatifs de leurs infrastructures et équipements, de leur main d'œuvre, de leur capacité d'innovation mais aussi de leur patrimoine. L'objectif est de devenir une « ville globale » ou une " ville de classe mondiale ».

Depuis 2000, les gouvernements municipaux disposent de compétences étendues en termes de développement économique ${ }^{10}$ et doivent agir en "gouvernement local développemental » permettant $d^{\prime}$ ' améliorer progressivement la situation sociale et économique des communautés et la fourniture de services de base à toutes [les] populations, et particulièrement les pauvres et les défavorisés " (Republic of South Africa, 2000, p. 1).

Par la promotion de l'« économie créative " censée stimuler la croissance économique et générer des emplois qualifiés et non qualifiés, The Fringe avait l'ambition initiale de combiner ces deux impératifs. Il a abouti à un projet hybride destiné uniquement à régénérer un quartier jusqu'à présent délaissé par les investisseurs: l'impératif développemental a été dilué au profit d'un investissement localisé. Le Projet urbain capetonien reste porté par une rationalité néolibérale : les investissements concentrés dans des espaces vitrines, dont le centre-ville, doivent permettre, à terme, aux populations les plus pauvres de bénéficier des fruits de la croissance économique et se répercuter sur les quartiers périphériques défavorisés. Une telle conception du développement "par ruissellement " est l'une des illustrations de la transition d'un urbanisme managérial à un urbanisme entrepreneurial (Harvey, 1989). Ce changement qualitatif du rôle de l'État local qui n'est plus que l'un des acteurs de la gouvernance urbaine résulte de la libéralisation économique impulsée à l'échelle nationale avec le programme Growth, Employment, and Redistribution de $1996^{11}$ et de la décentralisation réalisée en 2000 . L'implication croissante d'acteurs privés dans la gestion urbaine par le développement de partenariats public-privé et de groupes de pression est l'une des formes de l' « urbanisation du néolibéralisme » (Brenner et Theodore, 2002 ; Didier, Morange et Peyroux, 2012).

Afin d'identifier les rationalités qui sous-tendent ce projet de développement urbain, je me suis appuyé sur une analyse des discours des institutions prenant part à la coalition de croissance forgée à l'occasion, sur la littérature grise qu'elles ont produite et sur des entretiens avec les individus directement impliqués au sein de ces organisations. Au cours de ces entretiens, j'ai pu confronter le discours des institutions à celui des individus, identifier les convergences, les divergences et les négociations au sein de la coalition de croissance, ainsi que les rapports de domination au sein de la gouvernance urbaine du Cap. Le discours, étayé par des rationalités politiques et économiques, est en effet porteur de valeurs et de pratiques de domination.

\footnotetext{
${ }^{10}$ Avec le processus de décentralisation du début des années 2000, l'impératif de développement économique et social se décline à toutes les échelles de gouvernement (national, provincial et local).

${ }^{11}$ En 1994, le gouvernement Mandela lance le Reconstuction and Development Programme, une politique publique redistributive visant à surmonter les inégalités et le retard de développement engendrés par I'apartheid. L'adoption du programme Growth, Employment and Redistribution en 1996 constitue un tournant vers la libéralisation de l'économie, tournant renforcé en 1999 par l'élection de Thabo Mbeki, contesté au sein même de l'African National Congress (ANC, parti historique de la lutte contre l'apartheid, au pouvoir depuis les premières élections libres et multiraciales de 1994) pour sa vision néolibérale.
} 
Cette méthode met en évidence le rôle prépondérant d'un réseau très restreint d'élites urbaines non élues qui ont parfois travaillé pour plusieurs institutions au cours de leur carrière et qui détiennent le réel pouvoir de décision au sein de la gouvernance urbaine. Ces élites sont souvent impliquées dans plusieurs organisations (elles sont membres de plusieurs conseils d'administration) et entretiennent des relations interpersonnelles. Ce réseau enchevêtré constitue des passerelles entre les secteurs, parapublic et privé (Radovcic, 2011). Une approche par le réseau permet ainsi de saisir les relations de pouvoir diffuses, conflictuelles ou non, et d'identifier les acteurs dominants, qu'ils soient des organisations ou des individus.

Andrew Boraine est sans doute l'une des personnalités les plus emblématiques de ce réseau d'élites. Figure locale extrêmement respectée au Cap et dans tout le pays, et fils d'Alex Boraine, un ancien député de l'opposition progressiste sous l'apartheid, Andrew Boraine a lui-même milité contre le régime ségrégationniste au sein de l'United Democratic Front ${ }^{12}$. En raison de son activisme, il a effectué des peines de prison et a été assigné à résidence au début des années 1980. Pendant la transition démocratique, de 1991 à 1994, A. Boraine est directeur adjoint en charge du Gouvernement Local au Ministère du Développement Constitutionnel. Il participe alors à la rédaction de certains articles de la Constitution de 1996. Après avoir été City Manager ${ }^{13}$ de la municipalité de transition du Cap entre 1997 et 2000, il devient conseiller spécial auprès du Ministre du Gouvernement Local et Provincial en charge de la conception de stratégies de développement urbain et de politiques urbaines. II est l'artisan de l'établissement du réseau des neuf plus grandes métropoles sud-africaines, le South African Cities Network, dont il a été membre du conseil d'administration jusqu'en 2009. Andrew Boraine a été engagé pour participer à des missions de conseil sur la croissance économique et de développement de l'emploi sous l'égide de l'OCDE à Belfast, Derry et Barcelone. II a par ailleurs travaillé en collaboration avec différentes organisations internationales comme les Nations Unies, la Banque mondiale, le Gouvernement britannique, USAID (I'agence de coopération des Etats-Unis) ou encore l'Alliance des Villes ${ }^{14}$. Son expertise dans l'aménagement urbain et l'administration des villes est reconnue aussi bien nationalement qu'internationalement. Depuis 2003, il est directeur général du Cape Town Partnership (CTP), le puissant partenariat public-privé du centre-ville, créé en 1999, dont la mission est de revitaliser le centre-ville en capitalisant sur la centralité de l'espace et son exceptionnel patrimoine historique. Cette régénération urbaine s'est fondée sur la lutte contre la " criminalité et la crasse " entraînant un " nettoyage social " du centre-ville (Miraftab, 2007) tout en favorisant les investissements publics et privés et la conversion

\footnotetext{
12 L'United Democratic Front était l'une des organisations anti-apartheid les plus influentes dans les années 1980. Composé d'organisations étudiantes, de syndicats de travailleurs, d'églises et d'associations citadines (civics), I'UDF a mené des actions visant à rendre l'Afrique du Sud de l'apartheid ingouvernable tout en refusant de se lancer dans la lutte armée, à la différence de l'ANC.

${ }^{13}$ Le City Manager est le plus haut fonctionnaire dans l'organigramme municipal. II met en œuvre les politiques publiques décidées par le conseil municipal qui, lui, est élu.

14 L'Alliance des Villes est une coalition de Villes, d'Etats, d'organisations non gouvernementales et d'organisations internationales parmi lesquelles I'Union européenne, UN-Habitat, le Programme des Nations Unies pour le Développement et la Banque mondiale. Créée en 1999, elle a pour objectif de faciliter la mise en œuvre des programmes de développement urbain de la Banque mondiale. Elle promeut les «bonnes pratiques " de développement urbain et prône l'harmonisation de ces politiques à travers des approches standardisées comme les City Development Strategies (Stratégies de Développement Urbain).
} 
d'immeubles commerciaux ou de bureaux en immeubles résidentiels.

A. Boraine dispose sans doute de l'un des réseaux relationnels les plus développés du Cap. Ce réseau lui donne accès à des informations sur les orientations stratégiques sur la gouvernance urbaine capetonienne, sur les « best practices " développées en Afrique du Sud et à l'étranger et lui permet de mobiliser des acteurs publics comme privés autour des projets développés par le Cape Town Partnership. A. Boraine effectue un lobbying actif pour le compte du CTP aussi bien auprès des pouvoirs publics que du secteur privé, mais aussi du monde scientifique. Il siège au conseil d'administration de nombreuses organisations parapubliques et de groupes de pression, dispose de relais auprès du secteur privé ainsi qu'à la Municipalité du Cap dont il a été l'un des hauts fonctionnaires. II est l'initiateur de la création de la grande agence métropolitaine de développement urbain, l'Economic Development Agency ${ }^{15}$, qui devrait être lancée prochainement. Il est d'ailleurs pressenti pour en être le dirigeant. A. Boraine est en outre chercheur associé à l'African Centre for Cities ${ }^{16}$ (ACC), l'un des principaux laboratoires de recherche sur les dynamiques urbaines en Afrique du Sud, hébergé par la prestigieuse Université du Cap.

Alors que les milieux politique et scientifique avaient pu fortement décrier, à juste titre, son prédécesseur à la tête du Cape Town Partnership (Miraftab, 2007 ; Dubresson, 2008 ; Morange et Didier, 2008), Andrew Boraine et les orientations toujours néolibérales du CTP sont beaucoup moins questionnées aujourd'hui, du moins publiquement. II faut dire que $A$. Boraine a ajouté un volet social aux actions et à la communication du CTP ${ }^{17}$. Par ailleurs, sa proximité avec le milieu scientifique capetonien renforce encore l'énorme crédit dont il dispose en raison de son parcours personnel exemplaire. Ainsi, A. Boraine a participé, avec plusieurs chercheurs aujourd'hui associés à l'ACC, à la rédaction d'un article publié dans Urban Sudies (Boraine et al., 2006) promouvant un rapport du South African Cities Network (il était alors membre du conseil d'administration du réseau). Il propose sa vision, et celle du Cape Town Partnership pour le centre-ville du Cap dans un ouvrage scientifique publié par I'African Centre for Cities en 2010 (Pieterse, 2010). II participe, comme en février 2011, à des conférences sur le développement urbain au Cap en tant que chercheur de I'ACC. La tradition militante des universitaires sud-africains durant l'apartheid, y compris au Cap, explique sans doute le rôle d'acteur du développement urbain endossé par nombre de chercheurs locaux. II convient aussi de rappeler que le chantier de la construction de la ville post-apartheid offre des opportunités extrêmement stimulantes d'engagement personnel. Néanmoins, il me paraît dommageable que beaucoup de chercheurs en sciences sociales locaux travaillant sur Le Cap ne questionnent pas la prépondérance de ces élites urbaines et participent parfois à la diffusion et à la légitimation de la vision dominante, notamment à travers les travaux de l'African Centre for Cities.

Conduire des recherches au Cap appelle à adopter une posture critique à plusieurs titres. La

\footnotetext{
${ }^{15}$ La forme juridique de l'Economic Development Agency n'est pas encore arrêtée. Cette agence devrait être une association à but non lucratif financée en grande partie par la Province du Cap occidentale. Elle devrait permettre de stimuler l'investissement et de réduire le chômage dans la région métropolitaine.

${ }^{16}$ Ce laboratoire est d'ailleurs financé, entre autres, par le Cape Town Partnership.

17 Malgré ce tournant, le « nettoyage social » du centre-ville est toujours en cours (Radovcic, 2010). De nombreux articles de la presse locale (Cape Times et Cape Argus) relatent les cas de sans-abri arrêtés par les agents du Central City Improvement District (le Business Improvement District en charge du centre-ville dont l'action est coordonnée par le Cape Town Partnership) et " déposés » dans des quartiers périphériques.
} 
permanence des inégalités sociales héritées de l'apartheid rend une analyse en termes de classes pertinente. Ces classes socio-raciales sont des constructions sociales imposées durant l'apartheid mais toujours en partie opérantes aujourd'hui. Une telle analyse ne permet cependant pas d'appréhender le tournant libéral qu'ont pris les orientations des politiques publiques depuis 1996. Un réseau d'élites non élues impose sa conception néolibérale du développement au Cap : les interventions publiques (et l'argent public par là même) se concentrent sur des territoires déjà privilégiés dans la mesure où elles offrent de meilleurs retours sur investissement. Cette prépondérance des élites implique d'autant plus une démarche critique pour étudier les dynamiques urbaines au Cap qu'une partie des chercheurs en sciences sociales locaux contribue à la diffusion et à la légitimation de la vision dominante.

\section{Bibliographie}

Bond P. (2000) Elite transition: From apartheid to neoliberalism in South Africa, Durban, University of Natal Press.

Boraine A., Crankshaw O., Engelbrecht C., Gotz G., Mbanga S., Narsoo M., Parnell S. (2006) "The state of South African Cities a decade after Democracy ", Urban studies, vol. 43, $n^{\circ} 2$, pp. 259-284.

Brenner N., Theodore N. (2002) "Cities and the geographies of "actually existing neoliberalism" ", Antipode, vol. 34, n³, pp. 349-379.

Christopher A. J. (1994), The atlas of apartheid, Londres, Routledge.

City of Cape Town (2010) Draft Analysis of the Cape Town Spatial Economy, Le Cap.

Didier S., Morange M., Peyroux E. (2012) «The Adaptative Nature of Neoliberalism at the Local Scale: Fifteen Years of City Improvement Districts in Cape Town and Johannesburg ", Antipode, publié en ligne le 27 mars 2012, DOI: 10.1111/j.1467-8330.2012.00987.x

Dubresson A. (2008) «Urbanisme entrepreneurial, pouvoir et aménagement: les City Improvement Districts au Cap », in Dubresson A. et Jaglin S. (dir.), Le Cap après I'apartheid : gouvernance métropolitaine et changement urbain, Paris, Karthala, pp. 183-215.

Fauvelle-Aymar F.-X. (2006) Histoire de l'Afrique du Sud, Paris, Seuil.

Gervais-Lambony P. (2003) Territoires citadins : quatre villes africaines, Paris, Belin.

Guillaume P. et Houssay-Holzschuch M. (1998) « Du township à la ville... Nouveaux chemins de l'identité urbaine des Noirs sud-africains ", Géographie et Cultures, n² 28, pp. 47-59.

Harvey D. (1989) « From managerialism to entrepreneurialism: the transformation in urban governance in late capitalism », Geografiska Annaler, $n^{\circ} 71$, pp. 3-17.

Houssay-Holzschuch M. (1998) «Espaces métis, espace détruit, espace exemplaire : District Six, Cape Town », Géographie et Cultures, $n^{\circ} 25$, pp. 55-74.

Houssay-Holzschuch M. (2010) Vivre ensemble dans l'Afrique du Sud post-apartheid, Habilitation à Diriger des Recherches, Paris, Université Paris 1 Panthéon-Sorbonne, non publiée.

Leildé A. C. (2008) Changing identities in urban South Africa: an interpretation of narratives in Cape Town, thèse de doctorat, Stellenbosch, Université de Stellenbosch, non publiée. 
Lemanski C. (2007) "Global cities in the South: Deepening social and spatial polarisation in Cape Town », Cities, vol. 24, n 6, pp. 448-461.

McDonald D. A. (2008) World City Syndrome: Neoliberalism and inequality in Cape Town, New York, Routledge.

Miraftab F. (2007) " Governing post-apartheid spatiality: Implementing City Improvement Districts in Cape Town », Antipode, vol. 39, n 4, pp. 602-626.

Morange M. et Didier S. (2008) «Gouvernance sécuritaire dans les quartiers riches du Cap (1996-2006) : Temporalités et échelles de la normalisation post-apartheid ", in Dubresson A. et Jaglin S. (dir.), Le Cap après l'apartheid: gouvernance métropolitaine et changement urbain, Paris, Karthala, pp. 216-249.

Pieterse E. (dir.) (2010) Counter Currents: Experiments in Sustainability in the Cape Town Region, Le Cap, Jacana Media.

Republic of South Africa (2010) Municipal System Act, Pretoria.

Radovcic T. (2010) Les espaces publics à l'épreuve du tourisme: pratiques, représentations et gouvernance au Cap, mémoire de Master 1, Paris, Université Paris 1 Panthéon-Sorbonne, non publié.

Radovcic T. (2011) Entre compétitivité internationale et développement : la " ville créative » au Cap, mémoire de Master 2, Paris, Université Paris 1 Panthéon-Sorbonne, non publié.

Turok I. (2001) « Persistent polarisation post-apartheid? Progress towards urban integration in Cape Town ", Urban Studies, vol. 38, n 13, pp. 2349-2377.

Western J. (1981) Outcast Cape Town, Le Cap, Human \& Rousseau. 